\title{
SOME MOMENT RELATIONS FOR THE HIPP APPROXIMATION
}

\author{
By Jan DhaEne \\ Katholieke Universiteit Leuven \\ BJøRN SUNDT \\ University of Bergen \\ Nelson De PriL \\ Katholieke Universiteit Leuven
}

\begin{abstract}
In the present note we consider the Hipp approximation to the convolution of a finite number of probability distributions on the non-negative integers. It is shown that the moment up to and including order $r$ of the $r$ th order approximation are equal to the corresponding moments of the exact distribution. We also give a relation between the exact and approximated $(r+1)$ th order moments and indicate how similar relations can be obtained for higher order moments.
\end{abstract}

\section{KEYWORDS}

Hipp approximation, moments.

\section{PRELIMINARIES}

Let $f$ be the discrete density of a probability distribution on the non-negative integers. We shall denote the $j$ th order moment of $f$ by $\mu_{f}(j)$, the $j$ th order central moment by $\sigma_{f}(j)$, the $j$ th order cumulant by $\kappa_{f}(j)$, the probability generating function by $\rho_{f}$, the moment generating function by $\tau_{f}$, and the cumulant generating function by $\theta_{f}$, Thus we have

$$
\rho_{f}(s)=\sum_{x=0}^{\infty} s^{x} f(x) \quad \tau_{f}(s)=\rho_{f}\left(e^{s}\right) \quad \theta_{f}(s)=\ln \tau_{f}(s)
$$

$$
\begin{array}{rlrl}
\rho_{f}(s) & =\tau_{f}(\ln s)=\exp \left(\theta_{f}(\ln s)\right. & \\
f(x) & =\left.\frac{1}{x !} \frac{\mathrm{d}^{x}}{\mathrm{~d} s^{x}} \rho_{f}(s)\right|_{s=0} & & (x=0,1, \ldots) \\
\mu_{f}(j) & =\sum_{x=0}^{\infty} x^{j} f(x)=\left.\frac{\mathrm{d}^{j}}{\mathrm{~d} s^{j}} \tau_{f}(s)\right|_{s=0} & & (j=0,1, \ldots)
\end{array}
$$




$$
\begin{array}{ll}
\sigma_{f}(j)=\sum_{x=0}^{\infty}\left(x-\mu_{f}(1)\right)^{j} f(x) & (j=0,1, \ldots) \\
\kappa_{f}(j)=\left.\frac{\mathrm{d}^{j}}{\mathrm{~d} s^{j}} \theta_{f}(s)\right|_{s=0} & (j=0,1, \ldots)
\end{array}
$$

In particular we have that $\mu_{f}(0)=1$ and $\kappa_{f}(0)=0$.

In the present note we shall apply formal expansions like

$$
\begin{gathered}
\tau_{f}(s)=\sum_{j=0}^{\infty} \frac{\mu_{f}(j)}{j !} s^{j} \\
\theta_{f}(s)=\sum_{j=0}^{\infty} \frac{\kappa_{f}(j)}{j !} s^{j}
\end{gathered}
$$

without restricting to the situation where moments of all orders exist. Except for these formal expansions, it will always be silently assumed that moments, etc. that appear in displayed formulae, exist and are finite.

The moments can be evaluated from the cumulants by the recursion

$$
\mu_{f}(j+1)=\sum_{i=0}^{j}\left(\begin{array}{l}
j \\
i
\end{array}\right) \kappa_{f}(i+1) \mu_{f}(j-i), \quad(j=0,1, \ldots)
$$

which is easily obtained by (3), (4), and

$$
\tau_{f}^{\prime}(s)=\theta_{f}^{\prime}(s) \tau_{f}(s)
$$

From (5) we see that for any positive integer $r$, there is a one-to-one relation between the moments up to order $r$ and the cumulants up to order $r$; analogously there is a one-to-one relation between the central moments up to order $r$ and the moments up to order $r$. With formulations like "up to order $r$ " we shall always mean that $r$ is included

In the following we shall discuss approximations to probability distributions. As these approximations are not necessarily probability distributions themselves, we shall extend the concepts of moments, cumulants, and their generating function as defined above to more general functions. The recursion (5) still holds under this generalization. We shall only consider approximations for which the zero-th order moment is positive so that the zero-th order cumulants exists.

\section{THE HIPP APPROXIMATION}

Let $f_{1}, \ldots, f_{m}$ be the discrete densities of $m$ probability distributions on the nonnegative integers, and let $f$ denote their convolution. Then

$$
\theta_{f}(s)=\sum_{i=1}^{m} \theta_{f_{i}}(s)=\sum_{i=1}^{m} \ln \tau_{f_{i}}(s)=\sum_{i=1}^{m} \sum_{k=1}^{\infty} \frac{(-1)^{k+1}}{k}\left(\tau_{f_{i}}(s)-1\right)^{k} .
$$


The $r$ th order Hipp approximation $f^{(r)}$ to $f$ is defined by replacing the infinite summation in (6) with a finite summation containing only the first $r$ terms, that is,

$$
\theta_{f^{(r)}}(s)=\sum_{i=1}^{m} \sum_{k=1}^{r} \frac{(-1)^{k+1}}{k}\left(\tau_{f_{i}}(s)-1\right)^{k}
$$

From (1) and (2) we obtain

$$
f^{(r)}(x)=\left.\frac{1}{x !} \frac{\mathrm{d}^{x}}{\mathrm{~d} s^{x}} \exp \left(\theta_{f^{(r)}}(\ln s)\right)\right|_{s=0} . \quad(x=0,1, \ldots)
$$

Hipp (1986) gave an algorithm for recursive evaluation of $f^{(r)}$. Error bounds for the Hipp approximation have been studied by Hipp (1986), Hipp \& Michel (1990), De Pril (1989), and Dhaene \& De Pril (1994) for the case when $f_{i}(0)>\frac{1}{2}$ for $i=1, \ldots, m$.

\section{SOME MOMENT RELATIONS}

By introducing formal power series expansions in (6) and (7) we obtain

$$
\begin{aligned}
& \sum_{j=0}^{\infty} \frac{\kappa_{f}(j)}{j !} s^{j}=\sum_{i=1}^{m} \sum_{k=1}^{\infty} \frac{(-1)^{k+1}}{k}\left(\sum_{l=1}^{\infty} \frac{\mu_{f_{i}}(l)}{l !} s^{l}\right)^{k} \\
& \sum_{j=0}^{\infty} \frac{\kappa_{f(r)}(j)}{j !} s^{j}=\sum_{i=1}^{m} \sum_{k=1}^{r} \frac{(-1)^{k+1}}{k}\left(\sum_{l=1}^{\infty} \frac{\mu_{f_{i}}(l)}{l !} s^{l}\right)^{k} .
\end{aligned}
$$

By comparison of the coefficient of $s^{j}$ on both sides of the equality sign of (9) for $j=0,1, \ldots, t$, we see that $\kappa_{f^{(r)}}(0), \ldots, \kappa_{f^{(r)}}(t)$ exist and are finite if that is the case with $\mu_{f}(0), \ldots, \mu_{f}(t)$.

Subtracting (9) from (8) gives

$$
\sum_{j=0}^{\infty} \frac{\kappa_{f}(j)-\kappa_{f^{n}}(j)}{j !} s^{j}=\sum_{i=1}^{m} \sum_{k=r+1}^{\infty} \frac{(-1)^{k+1}}{k}\left(\sum_{l=1}^{\infty} \frac{\mu_{i}(l)}{l !} s^{l}\right)^{k} .
$$

As the lowest power of $s$ in the right hand expression of $(10)$ is $r+1$, we obtain

$$
\kappa_{f}(j)=\kappa_{f^{\prime r}}(j), \quad(j=0,1, \ldots r)
$$

that is, the cumulants up to order $r$ are exact for the $r$ th order Hipp approximation. As the moments up to order $r$ are uniquely determined by the cumulants up to order $r$, we obtain that also the moments up to order $r$ are exact; analogous we obtain that the central moments up to order $r$ are exact.

From the above we see in particular that for all $r$,

$$
\sum_{x=0}^{\infty} f^{(r)}(x)=\mu_{f^{(r)}}(0)=\mu_{f}(0)=1
$$


that is, the approximation sums to one like a probability distribution. Furthermore, in general the mean is exact for the Hipp approximation. This was also pointed out by Hipp (1986).

As we now know that the moments up to order $r$ are exact for the $r$ th order Hipp approximation, it is interesting to see how well this approximation approximates the $(r+1)$ the order moment. From $(10)$ we obtain

$$
\kappa_{f(r)}(r+1)=\kappa_{f}(r+1)+(-1)^{r+1} r ! \sum_{i=1}^{m} \mu_{f_{1}}(1)^{r+1} .
$$

Application of (11) and (5) gives that also

$$
\mu_{f^{(r)}}(r+1)=\mu_{f}(r+1)+(-1)^{r+1} r ! \sum_{i=1}^{m} \mu_{f_{i}}(1)^{r+1},
$$

and by expressing the central moments by the moments we obtain

$$
\sigma_{f^{(r)}}(r+1)=\sigma_{f}(r+1)+(-1)^{r+1} r ! \sum_{i=1}^{m} \mu_{f_{i}}(1)^{r+1} .
$$

Analogously it is possible to find relations between exact and approximated quantities of higher orders. However, then the relations will be more complicated.

Let us finally consider the special case $r=1$. Formula (12) gives

$$
\sigma_{f^{(1)}}(2)=\sigma_{f}(2)+\sum_{i=1}^{m} \mu_{f_{i}}(1)^{2}=\sum_{i=1}^{m}\left[\sigma_{f_{i}}(2)+\mu_{f_{i}}(1)^{2}\right] \text {, }
$$

that is,

$$
\sigma_{f^{(1)}}(2)=\sum_{i=1}^{m} \mu_{f_{i}}(2)
$$

This relation is not surprising as it is known that the first order Hipp approximation is equal to the ordinary collective compound Poissen approximation to the individual model, cf. Hipp (1986).

\section{REFERENCES}

DE PrIL, N. (1989). The aggregate claims distribution in the individual model with arbitrary positive claims. ASTIN Bulletin, 19, 9-24.

Dhaene, J. \& De PRIL, N. (1994). On a class of approximative computation methods in the individual model. Insurance: Mathematics and Economics, 14, 181-196.

HIPP, C. (1986). Improved approximations for the aggregate claims distribution in the individual model. ASTIN Bulletin 16, 89-100.

Hipp, C. \& Michel, R. (1990). Risikotheorie: Stochastische Modelle und Statistische Methoden. Verlag Versicherungswirtschaft e.V., Karlruhe. 
JAN DHAEne

Departement Toegepaste Economische Wetenschappen

Katholieke Universiteit Leuven

Huis Eighen Heerd

Minderbroederstraat 5

B-3000 Leuven

Belgium

BJøRN SUNDT

Department of Mathematics

University of Bergen

Allégaten 55

N-5007 Bergen

Norway

NELSON DE PRIL

Departement Toegepaste Economische Wetenschappen

Katholieke Universiteit Leuven

Naamsestraat 69

B-3000 Leuven

Belgium 